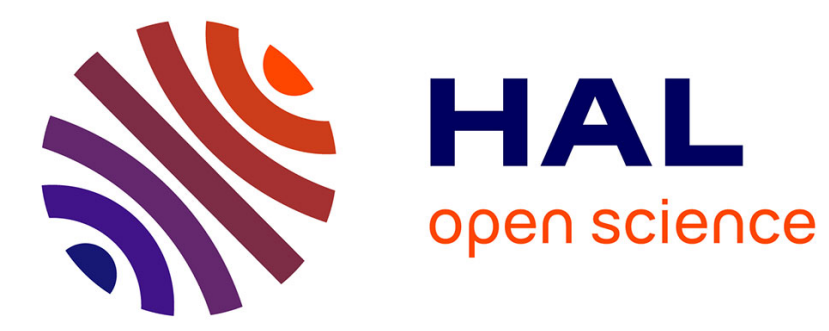

\title{
Vers un retour du politique dans le néo-institutionnalisme \\ Marc Smyrl
}

\section{To cite this version:}

Marc Smyrl. Vers un retour du politique dans le néo-institutionnalisme. Pôle Sud - Revue de science politique de l'Europe méridionale, 2005, La Turquie face à l'Europe, 23 (1), pp.115-130. 10.3406/pole.2005.1245. hal-01405308v2

\section{HAL Id: hal-01405308 \\ https://hal.science/hal-01405308v2}

Submitted on 19 May 2020

HAL is a multi-disciplinary open access archive for the deposit and dissemination of scientific research documents, whether they are published or not. The documents may come from teaching and research institutions in France or abroad, or from public or private research centers.
L'archive ouverte pluridisciplinaire HAL, est destinée au dépôt et à la diffusion de documents scientifiques de niveau recherche, publiés ou non, émanant des établissements d'enseignement et de recherche français ou étrangers, des laboratoires publics ou privés.

\section{(이) $\$$}

Distributed under a Creative Commons Attribution - NonCommercial - NoDerivatives $\mid 4.0$ 


\section{Vers un retour du politique dans le néo-institutionnalisme}

\section{Mr Marc Smyrl}

\section{Abstract}

The authors of this article propose a number of analytical elements complementary to historical institutionnalism. À number of these are drawn from the French school of public policy analysis, and its notion of the " référentiel », which here is revised and generalized. This allows a renewed emphasis on the role of actors at the heart of the analytical approach and heralds a return of the notion of politics, here defined as as the dynamic interaction of power and legitimacy in the context of an institutionalized decision-making framework in the study of the making and implemention of public policy.

\section{Résumé}

Les auteurs de cet article proposent plusieurs éléments analytiques complémentaires aux approches néoinstitutionnalistes et, en particulier, à l'institutionnalisme historique. Plusieurs d'entre eux sont issus de l'approche cognitive française des politiques publiques, en premier lieu la notion du référentiel, ici revue et généralisée. Ceci permet une réinsertion de l'acteur au cœur du dispositif analytique et d'un retour en force de la notion du politique, une dynamique associant autorité et légitimité dans le cadre institutionnalisé de prise de décision dans l'analyse de l'élaboration et de la mise en œuvre des politiques publiques.

\section{Citer ce document / Cite this document :}

Smyrl Marc. Vers un retour du politique dans le néo-institutionnalisme. In: Pôle Sud, n²3, 2005. pp. 115-130;

doi : https://doi.org/10.3406/pole.2005.1245

https://www.persee.fr/doc/pole_1262-1676_2005_num_23_1_1245

Fichier pdf généré le 29/03/2019 


\title{
Vers un retour du politique dans le néo-institutionnalisme
}

\author{
M. Smyrl et al. ${ }^{1}$ \\ CEPEL/Université de Montpellier 1
}

\section{Résumé/Abstract}

Les auteurs de cet article proposent plusieurs éléments analytiques complémentaires aux approches néo-institutionnalistes et, en particulier, à l'institutionnalisme historique. Plusieurs d'entre eux sont issus de l'approche cognitive française des politiques publiques, en premier lieu la notion du référentiel, ici revue et généralisée. Ceci permet une réinsertion de l'acteur au cour du dispositif analytique et d'un retour en force de la notion du politique, une dynamique associant autorité et légitimité dans le cadre institutionnalisé de prise de décision dans l'analyse de l'élaboration et de la mise en cuvre des politiques publiques.

The authors of this article propose a number of analytical elements complementary to historical institutionnalism. A number of these are drawn from the French school of public policy analysis, and its notion of the "refefentiel ", which here is revised and generalized. This allows a renewed emphasis on the role of actors at the heart of the analytical approach and heralds a return of the notion of politics, here defined as as the dynamic interaction of power and legitimacy in the context of an institutionalized decision-making framework in the study of the making and implemention of public policy.

\section{$\approx$ \\ Mots-Clés/Keywords}

Néo-institutionalisme, politiques publiques, référentiel

Neo institutionalism, public policies, refférentiel 
Cet article, première étape d'un projet collectif rassemblant des politologues européens et américains, propose une synthèse méthodologique alliant des éléments issus des néo-institutionnalismes avec ceux de "l'approche française " des politiques publiques. Dans ces deux milieux analytiques, l'étude du changement dans les politiques et de la formation des préférences des acteurs sont à l'ordre du jour (Hall, 2004; Thelen, Streeck, 2004; Muller, 2005). Notre réflexion s'inscrit dans cette lignée, et cherche d̀ $y$ apporter quelques éléments complémentaires. Il s'agit pour nous de réintroduire de manière plus directe le politique dans l'analyse institutionnelle. De cette façon, nous entendons les relations complexes entres idées, acteurs et pouvoir. Le traitement de cette problématique est, à notre avis, justement l'une des grandes forces de l'approche française, par rapport aux néoinstitutionnalismes d'origine anglo-américain. (Jobert, 1995; Muller, 1995). Elle permet de prendre des distances aussi bien vis-à-vis du structuralisme parfois excessif de ces derniers que de l'imprécision qui accompagne trop souvent "l'approche par les idées" (Muller, 2005). Ce ne sont pas les idées, les institutions ou les intérêts qui font les politiques publiques (Surel, 1998), mais les acteurs, des acteurs qui subissent bien sûr toutes ces contraintes, mais qui conservent néanmoins une certaine liberté d'action et qui, dans leurs actions quotidiennes, recherchent autant le pouvoir ou le respect de normes, que la vérité.

Ce monde du politique, et nous devons d'entrée de jeu y insister, ne se limite pas au milieu " politicien " des partis et des élus. Le politique, pour nous, n'est ni un milieu, ni une profession, mais une dynamique impliquant un ensemble d'acteurs et d'actions orientés vers la conquête et l'exercice du pouvoir. Une dynamique que l'on peut retrouver dans les partis et les parlements, mais aussi dans les administrations, les syndicats ou les entreprises, et qui associe autorité et légitimité dans le cadre institutionnalisé du processus de prise de décision.

Dans un premier temps, nous ne cherchons pas à produire un modèle déductif, susceptible d'une preuve empirique. Nous proposons plutôt un cadre analytique à l'intérieur duquel des modeles pourraient éventuellement se situer. A ce stade de la réflexion, la valeur de ce cadre réside principalement dans deux éléments. D'abord, la production d'un vocabulaire analytique 
commun qui autorise la comparaison et la discussion d'un grand nombre de projets très divers, aussi bien dans leurs méthodes que dans leurs objets; ensuite, une grille d'analyse, tout à la fois systématique et souple, adaptée à l'étude comparée des politiques publiques dans des conditions très diverses. La finalité de cette contribution est donc de fournir les premiers fondements théoriques permettant d'étayer l'un et l'autre.

Notre point de départ se trouve dans les " néo-institutionnalismes " de la littérature anglophone, et tout particulièrement dans l'approche dite " historique " (Hall, Taylor, 1997). Deux étapes principales marqueront ensuite le chemin menant à la réintroduction du politique dans l'institutionnalisme historique. Le premier est une application de certains principes et notions empruntés à l'école " française " des politiques publiques (Jobert, Muller, 1987 ; Faure, Pollet, Warin, 1995). Prenant ces apports en compte, nous devrons alors reformuler, avec certains changements, les éléments issus de ces sources diverses dans le but de proposer une nouvelle grille d'analyse du politique.

\section{Les néo-institutionnalismes en question}

Les approches analytiques regroupées sous le sigle des " néo-institutionnalismes" représentent une avancée importante dans l'étude des phénomènes politiques et une rupture avec les approches pluralistes anglosaxonnes, tout comme avec l'institutionnalisme positiviste français (Smyrl, 2002).

\section{Apports des néo-institutionnalismes}

La contribution essentielle des néo-institutionnalismes en science sociale a été de remettre en question en même temps le monisme abusif des approches systémiques en sociologie et le réductionnisme excessif des théories caractérisées par l'individualisme méthodologique absolu. Dans les deux cas, les institutions sont interposées entre le niveau de l'individu et celui de la société. En ce qui concerne l'étude de l'État et de ses politiques publiques, les « nouveaux institutionnalismes " permettent en même temps l'ouverture de la "boîte noire " de l'État et la reconnaissance de ce dernier comme un acteur (ou un ensemble d'acteurs) à part entière, jouissant d'une certaine autonomie vis-à-vis de la société.

Contre le caractère fonctionnaliste des modèles systémiques, le nouvel institutionnalisme dans sa version "sociologique " met en avant le rôle de la "cérémonie " et même de la "mythologie " dans une grande partie de l'action sociale (Meyer, Rowan, 1977 ; DiMaggio, Powell, 1991). Les vecteurs de cette rationalité axiologique ne sont ni les individus, ni les classes socio-économiques, ni la société tout entière, mais les composantes organisées de la société: professions, services publics, programmes, etc. ${ }^{2}$ Pour chacune de ces organisations, la rationalité axiologique forme un cadre institutionnel qui peut, dans certains cas, primer sur son but " rationnel ". Le cadre institutionnel fournit à l'organisation sa légitimité sociale. Dans la société moderne, la rationalité ellemême forme une partie de la mythologie légitimant les organisations et leurs structures.

Pour certains chercheurs en science politique, les principes de l'institutionnalisme sociologique se sont révélés d'une grande utilité pour expliquer la production et, surtout, la reproduction des politiques publiques. Pourquoi la politique ferroviaire dans l'Europe du XIX' siècle prit-elle des formes différentes d'un pays à un autre, mais 
dans chaque cas une forme semblable à la politique préalable de développement du transport fluvial dans le pays en question (Dobbin, 1994) ? Pourquoi les régions de l'Italie du centre et du nord mettent-elles en évidence des normes " civiques " de gouvernement, quel que soit le régime au pouvoir à Rome, et en contraste avec la tradition clientéliste tout aussi persistante du Mezzogiorno (Putnam, 1993) ? La reproduction des institutions sociales et la formation par ces mêmes institutions des préférences individuelles - y compris en ce qui concerne le monde politique - est avancée comme apportant une réponse.

La notion d'institution est également mobilisée, dans un contexte très différent, dans un effort de repositionnement de l'économie politique néo-libérale. Le fonctionnement theorique de la dynamique de marché donnerait lieu, si elle agissait pleinement, à un niveau d'homogénéité et d'efficacité hors de proportion avec ce qui peut réellement s'observer. La réponse théorique, proposée par la " nouvelle histoire économique " de $\mathrm{D}$. North, se trouve dans un mécanisme empêchant l'efficacité, le coût des transactions, et une solution sociale partielle à ce problème : les institutions (Noth, Thomas 1973; North, 1990). Si tout échange, y compris l'acquisition d'information, est coûteux, et donc soumis à des contraintes budgétaires, certains échanges intéressants n'auront pas lieu, et le résultat global restera en deçà du niveau optimal. C'est en facilitant l'échange dans certains cas, mais pas dans tous, que les institutions - règles et normes acceptées par les agents économiques - rendent à l'échange une partie de l'efficacité que le coût des transactions lui a enlevée.

Dans ce contexte, les règles formelles prennent une grande importance, et ceci pour les relations politiques tout autant qu'économiques. C'est par rapport à ces règles, et aux opportunités différenciées qu'elles offrent aux stratégies rationnelles des acteurs, que les adeptes de l'institutionnalisme des choix rationnels expliquent les actions des militants à l'intérieur d'un parti, des parlementaires à l'assemblée nationale de la $V^{e}$ République ou des chefs d'état, membres du Conseil Européen (Tsebelis, 1990 ; Huber, 1996; Moravcsik, 1998).

\section{Critique des néo-institutionnalismes}

Si différents dans leurs origines, les institutionnalismes " sociologiques " et " des choix rationnels" partagent certaines faiblesses de premier ordre. Chacun de son coté sous-entend une vision de l'acteur singulièrement appauvrie par rapport aux développements de la philosophe politique contemporaine. Ni l'un ni l'autre ne nous offrent une explication satisfaisante de l'origine première des préférences ou de leur éventuelle remise en question. Tous les deux, finalement, font la part trop belle à certaines variables structurelles, privant les acteurs de toute possibilité de choix réel.

La pauvreté philosophique du débat institutionnaliste mérite d'être plus souvent signalée - et déplorée. Les écoles " rationalistes " tout comme " sociologiques " en sont restées à des modèles depuis longtemps dépassés. L'approche rationaliste a toujours tendance à accepter une notion simpliste de l'utilitarisme datant de la génération de Bentham, un utilitarisme qui ne permet aux acteurs que la maximisation de biens préexistants - et non-théorisables ${ }^{3}$. L'institutionnalisme dit sociologique, commet l'erreur inverse, adoptant une forme naïve du modèle communautarien le plus extrême, qui voudrait voir tout acteur 
comme étant entièrement défini par son environnement social et institutionnel, incapable de percevoir de manière objective et, encore moins, d'évaluer ou de re-évaluer les préférences qui en résultent ${ }^{4}$. Ce débat stérile a, depuis longtemps déjà, été abandonné par une nouvelle génération de penseurs politiques. Rawls le libéral (1993), Sandel le républicain " unitaire "(1996), Kymlicka le défenseur des cultures minoritaires (1989), sont tous d'accord pour accorder à l'acteur moral une capacité de choix, d'évaluation et de re-évaluation, face aux projets moraux, mais aussi pratiques, offerts par un environnement institutionnel complexe et souvent contradictoires.

Ce constat philosophique se reporte au niveau analytique. Conçues pour expliquer la continuité, les approches institutionnalistes ont toutes les difficultés à expliquer origines et changement - qu'il s'agisse des préférences individuelles, des normes institutionnelles ou des politiques publiques concrètes. Ainsi, March et Olsen, dans une étude classique qui prétend porter sur les réformes, en arrivent à la conclusion que celles-ci sont pratiquement impossibles, et que la vie politique se résume à une oscillation permanente entre vision " agrégatives" et " intégratives " du gouvernement (March, Olsen, 1989). Pour l'institutionnalisme des choix rationnels, le changement est en principe moins problématique, puisqu'il est le résultat de " chocs " extérieurs et non-théorisés. Le prix analytique de cette flexibilité, malheureusement, est que les origines du changement, et des préférences - le cour même de la chose politique - ne peuvent pas être problématisées. Elles doivent être traitées de manière ad hoc, au mieux par l'observation empirique et au pire par des modifications théoriques $e x$ post.
De toutes les lacunes des modèles institutionnalistes, la plus grave, pour nous, est cette perte de vue du politique. Par là, nous entendons les choix réels, non déterminés à l'avance par la structure des institutions ou par une vision étroite de la rationalité, portant, non seulement sur les moyens, mais aussi sur les fins des politiques publiques. Nous constatons que les modèles inspirés des choix rationnels n'autorisent à leurs acteurs que des choix entre stratégies - et ne permettent jamais la remise en question des préférences pré-stratégiques. Quant aux approches " sociologiques ", le choix disparaît entièrement face à un déterminisme social et historique auquel aucun acteur ne peut espérer échapper.

Comme nous l'avons indiqué plus haut, nous estimons que ce structuralisme excessif ne rend pas pleinement compte de la dynamique qui mène, des idées et des intérêts, aux politiques publiques concrètes qui forment l'objet ultime de notre analyse. Ainsi que nous venons de le voir, les premiers institutionnalismes ne fournissaient aucun outil pour analyser les choix, non pas entre moyens, mais entre fins.

Les lacunes principales des modèles des nouveaux institutionnalismes dessinent en creux les contours de l'approche que nous désirons développer ici. Le retour du politique passe obligatoirement par une remise en scène de l'acteur et de l'action. Les acteurs, nous en sommes bien conscients, n'ont pas une liberté totale dans leurs choix. Ces choix n'en sont pas moins réels. Il s'agit donc de s'intéresser, de près et en même temps, au mécanisme du choix et à l'origine des options parmi lesquelles l'acteur est appelé à choisir. Une deuxième génération de l'institutionnalisme apporte certains éléments utiles à ces fins. 


\section{Les néo-institutionnalismes de} seconde génération: " Historique " et * acteur-centré *

Il s'agit ici d'un groupe d'auteurs, extrêmement hétérogènes, regroupés sous la bannière soit de "l'institutionnalisme historique ", soit de "l'institutionnalisme acteur-centré ". Parmi leurs travaux, nous trouvons des éléments de grande valeur, mais aucune véritable vision d'ensemble commune qui servirait à définir une approche généralisable.

Pour expliquer le changement dans un monde où les préférences sont, au moins en partie, endogènes, certains évoquent le rôle indépendant des idées. Pour Hall, par exemple, les vecteurs du changement en politique économique sont d'abord des intellectuels, essentiellement des professeurs et journalistes, qui animent ce qu'il qualifie de " marché extérieur d'idées" (1992): " extérieur " parce qu'en dehors des circuits traditionnels des fonctionnaires de la finance publique; " marché " parce que les théoriciens ne peuvent offrir seulement que leurs marchandises intellectuelles; il leur faut trouver un " acheteur ". Cet " acheteur ", dans le cas du monétarisme, ils le trouveront avec Mrs Thatcher, qui s'en servira comme arme de choc dans le triple combat politique qu'elle mène, dans un premier temps, contre la vieille garde de son propre parti, puis, une fois son autorité établie, contre ses ennemis politiques et sociaux : le parti travailliste et les syndicats.

Une autre tentative d'explication des origines et de l'évolution d'une politique publique peut être trouvée dans les travaux de T. Skocpol sur la "préhistoire " de l'État providence aux États Unis (1992) ${ }^{6}$. En suivant le cheminement de la politique d'assistance aux anciens combattants de la guerre de sécession ainsi que de l'action maternaliste de l'État américain, Skocpol met en valeur une dynamique complexe du changement, et des relations entre la politique publique et les intérêts sociaux. En s'appuyant sur de multiples exemples, elle démontre que les atouts, aussi bien politiques (lobby, réseaux d'alliances) qu'idéologiques à un moment donné, peuvent devenir des handicaps majeurs dans la période suivante - quand le système des pensions se trouve assimilé à la corruption.

La question de l'idéologie, son importance mais aussi sa production, sont au centre des derniers travaux de P. Katzenstein (1996). Prenant l'exemple de la reconstruction politique de l'Allemagne et du Japon après la Seconde Guerre mondiale, Katzenstein observe la mise en place par les élites politiques de ces deux pays de nouvelles bases idéologiques adaptées à la situation de leurs pays. Dans les deux cas, il montre que le choix de la nouvelle idéologie dominante n'a pas été imposé de l'extérieur (ni par les alliés, ni par les conditions " objectives" de la nouvelle situation). Au contraire, elle a été l'enjeu d'un combat politique acharné à l'intérieur de l'élite allemande et japonaise.

Ces travaux sont d'une grande importance. L'idéologie, variable exogène nonthéorisée dans les modèles des choix rationnels, est ici rattachée directement au politique. Les idées ne sont plus simplement des " sentiments partagés " dont les origines sont floues, comme dans beaucoup de modèles sociologiques, mais bien des produits directs des luttes de pouvoir et d'influence. Il resterait à généraliser dans ce domaine une méthode équivalente à celle de Skocpol, pour explorer non seulement la genèse mais aussi la transformation et la mort des idéologies. 
Dans un tout autre registre, celui des choix rationnels et de la théorie des jeux, le modèle de "l'institutionnalisme acteur-centré " représente lui aussi un pas en avant de première importance (Scharpf, 1997 ; Scharpf, Mayntz, 2001). Un premier apport de cette approche est son insistance sur l'aspect stratégique de l'élaboration des politiques publiques. Ce ne sont pas les préférences, les stratégies ou les choix d'un seul acteur qui déterminent les résultats, mais l'effet croisé des actions de tous les acteurs concernés.

Notant que les acteurs en politiques publiques sont, plus souvent, des collectivités (doté de plus ou moins d'organisation interne) que des personnes physiques, l'institutionnalisme " acteur-centrique " explore les possibilités et les limites de leurs actions selon leurs capacités internes et leurs éléments de rationalité (instrumentale, mais aussi cognitive). Cette action se déroulant dans un champ institutionnel, plus ou moins développé, Scharpf analyse les modalités d'action stratégique, allant de la négociation en absence d'institution à la décision hiérarchique - fortement institutionnalisée. En ce qui concerne les préférences et les motivations pré-stratégiques des acteurs, en revanche, Scharpf ne dit rien, remettant ces questions aux travaux empiriques. C'est justement sur ce point que l'approche française s'avère le plus utile.

\section{La variante française de l'analyse des politiques publiques}

Même les versions les plus sophistiquées de l'institutionnalisme des choix rationnels, telle l'approche de Scharpf, laisse volontairement à l'écart la question de l'origine des préférences. La mise en valeur de l'outillage descriptif et analytique ainsi développé demande donc un travail en amont pour savoir ce que veulent vraiment les acteurs. Les approches " historiques ", de leurs côtés, cherchent à répondre à cette question, mais se limitent jusqu'à présent à des études de cas non généralisées. Par contre, c'est l'école française des politiques publiques, celle de L'État en Action, qui nous permet de poser des jalons pour une discussion fertile.

\section{Apports du modèle de L'État en Action}

L'apport essentiel de l'approche française des politiques publiques est un traitement proprement politique et dynamique du rôle des idées et de leurs relations avec le pouvoir politique. Avec la notion de "référentiel ", ces chercheurs ont mis en place, de manière systématique, un modèle de relation entre idées, intérêts et pouvoir que certains chercheurs de "l'institutionnalisme historique", tels P. Hall ou P. Katzenstein, ont cherché de manière ad hoc à décrire dans les cas empiriques qu'ils ont traités. En insistant sur la problèmatisation des relations entre le référentiel global et les référentiels sectoriels, et en proposant un vocabulaire et un cadre analytique pour leurs travaux, Jobert et Muller offrent un outil fort utile pour expliquer le décalage, souvent observé, entre les politiques publiques entreprises dans tel ou tel secteur, et « l'air du temps » qui oriente la politique générale d'un gouvernement.

Un grand avantage du modèle français, par rapport à la plupart des modèles institutionnalistes, est que le référentiel offre, non seulement une grille cognitive, mais aussi une théorie de l'action. Il contient non seulement des normes, mais aussi des algorithmes pour la production des politiques publiques (Muller, 1995). Dans les écrits de B. Jobert et de P. Muller ultérieurs à L'État 
en Action, nous trouvons également une analyse particulièrement approfondie du couple idées-pouvoir. Pour Jobert (1995, p. 5), " la négation récurrente du politique " est un point de faiblesse de la sociologie des organisations. Muller (1995, p. 164), quant à lui, met l'accent sur le couple « champs intellectuel/champs de pouvoir. "S'appuyant sur la notion "d'hégémonie " dans le sens que donne à ce mot Gramsci ${ }^{7}$, il insiste sur l'interdépendance des sphères cognitives et politiques: "C'est parce qu'il définit le nouveau référentiel qu'un acteur prend le leadership du secteur en affirmant son hégémonie mais, en même temps, c'est parce que cet acteur affirme son hégémonie que sa vision du monde devient peu à peu la nouvelle norme " (Muller, 1995, p. 165).

Ce passage illustre également un dernier aspect de l'approche française des politiques publiques: le fait que les " idkes " ne sont pas des esprits désincarnés mais doivent être portées par des individus ou des groupes ${ }^{8}$. C'est le pouvoir politique de ces vecteurs, autant que le contenu de leurs idées, qui détermine les résultats (Joana, 2002).

\section{Critique du modèle de l'État en Action}

Ceci dit, il ne s'agit pas ici de reprendre " clef en main " le modèle de Jobert et Muller. Tout d'abord, celui-ci a connu une évolution importante depuis son introduction: aussi bien ses auteurs, qu'une génération de leurs étudiants et collègues, ont élaboré et également transformé le modèle (Hassenteufel, Smith, 2002; Muller, 2004; Jobert, 2004). Il n'existe donc pas une référence unique. Face à cette nébuleuse théorique néanmoins, nous tenons à marquer trois départs essentiels.

Une des critiques classiques porte sur le contenu même de l'approche française. La notion de "référentiel global ", en particulier, nous semble discutable. Il existe bien des " paradigmes " dominants pour de vastes ensembles d'activité politique tels que l'économie, la défense nationale, la politique sociale, etc. Cependant, invoquer ici un " référentiel global " unique ne nous parait pas évident. Cela interdirait, en particulier, l'analyse fructueuse des frontières souvent floues et contestées entre ces ensembles - économie et défense nationale, par exemple - et laisse entière la question de l'origine de ce référentiel global.

La distinction, entre un cadre cognitif général et d'autres plus particuliers à des activités données, est une des grandes forces de l'approche française, et nous n'entendons pas l'abandonner. La notion de "secteur ", par contre, ne doit pas constituer une référence automatique à un néo-corporatisme, pour partie dépassé; les frontières de nombre de secteurs, naguère encore bien défendus, apparaissent aujourd'hui poreuses à travers des politiques de plus en plus transversales.

Une dernière critique, finalement, n'est qu'un appel à la généralisation. Ouvrage français analysant les politiques publiques de l'état français, L'Etat en Action fait la part belle à certains acteurs, les hauts fonctionnaires, par exemple, dont le rôle, incontesté en France, ne se retrouve pas automatiquement ailleurs. Il s'agit ici de conserver l'approche analytique, tout en gardant la possibilité de chercher des acteurs équivalents, mais pas toujours identiques, dans d'autre cas.

A l'issue de ce que nous venons de dire, nous maintiendrons l'utilisation systématique des outils et du vocabulaire de l'institutionnalisme, y compris des choix rationnels, et de la théorie des jeux. Le modèle français, même avec les aménagements proposés ci-dessous, est un complément, et non un substitut, aux approches institutionnalistes. Situé en amont, il permet d'analyser et de théoriser le contenu cognitif et axiologique de l'action stratégique. 


\section{Pour une réintroduction du politique dans le néo-institutionnalisme}

Nous privilégions la réintroduction du " politique " dans notre approche et ceci pour plusieurs raisons. Tout d'abord, nous l'avons évoqué plus haut, pour insister sur la réalité des choix, mais aussi pour souligner l'importance accordée aux relations de pouvoir, d'influence et d'autorité. L'apport le plus évident de l'approche par le politique est celui invoqué au tout début de cet article. Notre approche synthétique articule la richesse cognitive du modèle du référentiel, au pouvoir analytique d'une version généralisée de la théorie des jeux. Nos critiques des approches françaises et anglophones laissent transparaître, en revanche, l'intérêt de certains éléments plus innovants.

Au cœur de ce dispositif, se trouvent les notions de "cadre d'ensemble cognitif " et de " modèle programmatique ". C'est en grande partie dans la dynamique et les contradictions entre ensembles et programmes que nous retrouvons la possibilité de choix et d'action. Cette dynamique est complétée par certains choix se situant en marge de la rationalité (axiologique tout comme instrumentale).

Par " ensemble cognitif " nous entendons un vaste champ d'action régi par un ensemble cohérent de définitions axiomatiques, aussi bien normatives que positives'. L'économie en est l'exemple, tout comme la défense nationale ou la médecine. La frontière entre un ensemble et un autre peut être floue et contestée (entre le système économique et celui de la protection de l'environnement, par exemple). Ce qu'apporte un cadre d'ensemble cognitif est une description complète de l'ensemble en question, partant de proposition axiomatique (par exemple: l'épargne est égale à l'investisse- ment ou la valeur n'a d'autre source que le travail) et construisant autour d'elle un ensemble de théorèmes explicatifs.

Un ensemble, peut être plus ou moins extensif. Certains peuvent même être qualifiés "d'impérialiste " ayant une vocation à l'expansion jusqu'à parfois prétendre " expliquer " la vie entière - ce qui est le cas pour certains cadres économiques, par exemple. Ceci dit, et tout en notant l'intérêt analytique de ces prétentions, nous préférons la métaphore des "sphères " de M. Walzer (1985) ${ }^{10}$. Tout comme la pluralité de sphères de justices dans l'analyse de ce philosophe, nous nous attendons à trouver une pluralité d'ensembles cognitifs dans le monde du politique, chacun avec ses bases axiomatiques et sa rationalité particulière (Joana, Smith, 2004; Genieys, Michel, 2004).

Plusieurs cadres peuvent se disputer l'hégémonie sur un même ensemble (économie libérale, marxiste, etc). De même, l'appartenance d'une politique publique concrète à tel ou tel ensemble n'est pas toujours évident, surtout en présence d'ensembles « impérialistes " ". Les tensions dynamiques ainsi créées, nous le verrons plus bas, fournissent aux acteurs autant d'opportunités de choix (Meyer, Rowan, 1977).

Implicitement, tout cadre est normatif, puisque tous prétendent offrir une description de la vérité objective. Toute ligne de conduite peut ainsi être jugée naturelle - et donc légitime - ou contre-nature. Un ensemble est trop vaste, néanmoins, pour engendrer une véritable théorie de l'action politique. C'est au niveau inférieur, celui du programme de politique publique, qu'il faut chercher celle-ci.

C'est au niveau du programme de politique publique que se situe l'action concrète et quotidienne de l'État. Les politiques de protection sociale, d'armement ou de commerce 
extérieur, pour ne citer que des exemples repris ci-dessous, sont des programmes de politiques publiques. C'est à ce niveau que nous nous attendons à trouver une organisation institutionnalisée, un budget, des résultats vérifiables. C'est à ce niveau que se trouve le savoir-faire technique.

Ce savoir-faire n'a de sens, néanmoins, que dans un cadre plus vaste, celui de l'ensemble cognitif. Si le niveau programmatique porte réponse aux questions de type "comment? " c'est au niveau de l'ensemble qu'il faut chercher la réponse aux questions de type "pourquoi? " Ce sont les données normatives, voire philosophiques, d'un système économique qu'il s'agisse d'un système libéral, keynesien, ou socialiste - qui donnent leur sens, leur légitimité, à des programmes de commerce extérieur, de politique monétaire, ou d'emploi.
Vu notre critique du structuralisme excessif de la plupart des modèles institutionnalistes, il est évident que nous ne voyons ni dans les cadres d'ensemble cognitif, ni dans les modeles programmatiques, une explication complète de l'action et des choix des acteurs. La pluralité des cadres comme des modèles en est une première raison. Un cadre peut bien, de manière objective, être logiquement complet en lui-même, mais rien n'assure qu'un acteur, de son propre point de vue subjectif, se situe entièrement à l'intérieur de ce cadre, à l'exclusion de tout autre. En plus, comme nous le verrons plus bas, il existe des situations dans lesquelles cadres et modèles n'offrent que des guides imparfaits et incomplets.

L'approche par le politique des institutions, dans la présentation générale que nous lui donnons ici, ne peut être qu'une

Dans son ensemble, notre approche peut se résumer par la série de propositions suivante:

I. Les politiques publiques représentent les résultats conjugués (joint outcomes) de l'action stratégique des acteurs, prenant en compte les contraintes imposées par les structures institutionnelles ou les systèmes d'idées.

II. Les préférences des acteurs, y compris leurs orientations en interaction vis-à-vis d'autres acteurs, sont influencées par le cadre d'ensemble cognitif et le modèle programmatique applicable.

a. L'imposition d'un nouveau cadre d'ensemble cognitif ou d'un nouveau modèle programmatique est une action de pouvoir politique.

b. L'harmonisation des modèles programmatiques avec les cadres d'ensemble cognitifs est le rôle d'élites particulières, les médiateurs capables de traduire et d'exprimer les éléments de cadres et de modèles à l'usage des acteurs concernés. En leur absence, les incohérences entre ces deux niveaux peuvent subsister.

III. Les stratégies par lesquelles les acteurs poursuivent leurs préférences subissent les contraintes de leur structure interne (dans le cas d'acteurs collectifs) et de la structure des règles institutionnelles à travers lesquelles ils agissent. L'imposition de nouvelles règles institutionnelles est une action de pouvoir politique.

IV.Les mécanismes de dépendance envers le sentier parcouru (path dependence) peuvent permettre aux règles, aux modèles et aux cadres de survivre aux conditions de leur création. Ces structures peuvent ainsi contraindre des acteurs non impliqués dans leur création ou justification d'origine.

V. Quand un ou plusieurs des éléments des propositions II ou III sont ambigus ou instables, l'occasion de " méta-choix " se présente aux acteurs.

VI. Les méta-choix auront une influence durable sur les résultats des politiques publiques dans la mesure où ils sont institutionnalisés en devenant eux-mêmes des bases de règle, de modèle ou de cadre. 
grille d'analyse. Elle fournit les éléments nécessaires à la description disciplinée des politiques publiques. C'est à partir de ces descriptions et à l'intérieur de ce cadre que des modèles prédictifs peuvent être élaborés.

\section{Politique et institutions en interaction}

La grille proposée ci-dessus permet d'analyser les politiques publiques, l'action concrète des États, autour des interactions entre le politique et les institutions. Notre approche n'est certainement pas la seule à afficher cette ambition. Nous estimons néanmoins que pour trois types de questions, l'approche par le politique serait particulièrement bien adaptée. Il s'agit tout d'abord du problème évoqué dans l'encadré ci-dessus, de la possibilité de conflit entre ensembles cognitifs ou entre un programme et "son" ensemble. Une seconde dimension analytique se trouve dans la distinction entre changements de préférence et changements de stratégie. Enfin, nous insistons sur l'importance des choix imparfaitement rationnels.

\section{Conflits entre niveaux : cadres d'ensembles cognitifs et modèles programmatiques}

Modèles et cadres ayant chacun une existence intellectuelle et institutionnelle relativement autonome, les possibilités de conflits sont nombreuses. Plusieurs cas de figures sont envisageables.

Il se peut, grâce à la continuité du personnel et des procédures, que le modèle soit plus stable que le cadre. La dynamique de path dependence se renforce en fonction de la présence de " densité institutionnelle " et des procédures alliant complexité technique et manque de transparence (Pierson, 2000,
2004). C'est au niveau des programmes, beaucoup plus qu'à celui des cadres cognitifs, que nous trouvons ces caractéristiques. Comme le montre l'analyse des conditions de professionnalisation des armées françaises et britanniques (Joana, 2004). Il est possible que les réponses aient la vie plus dure que les questions comme l'atteste bien l'évolution des politiques régionales de l'union européennes (Behrens, Smyrl, 1999).

Une autre dynamique, menant à un résultat semblable, est celle de l'ensemble cognitif " impérialiste " tentant d'annexer des nouveaux programmes. La question posée par ce cas est de savoir si de nombreux programmes, jadis régis par des cadres autonomes, par exemple les politiques de protection sociale, $d$ 'assurance maladie ou encore d'armement, sont devenus, dans le courant des années 1990, autant de "sousensemble " de la nébuleuse économique, soumise à un même et unique cadre d'ensemble hégémonique (Genieys, Hassenteufel, 2001 ; Genieys, 2005).

Un avantage du remplacement de la notion de référentiel global par celle de cadre d'ensemble cognitif est la possibilité de traiter le problème de compétition entre cadres. L'ensemble dans lequel un programme donné doit être placé n'est pas toujours évident. La lutte contre les stupéfiants, pour reprendre un exemple cité ci-dessus, relève-t-elle de "l'ensemble " de la santé publique, de la police ou de la défense nationale? Beaucoup dépend de la réponse à cette question - qui ne peut même pas être posée dans le contexte du modèle français des politiques publiques (Joana, Smith, 2004; Genieys, Michel, 2004 ${ }^{12}$ ).

Une dernière illustration de la dynamique de conflits entre niveaux se trouve lors de l'application d'un modèle programmatique, 
en dehors de son cadre d'ensemble cognitif d'origine (dans le contexte d'un système fédéral ou de l'Union Européenne, par exemple, ou d'un passage entre les secteurs public et privé.) La mise en œuvre des politiques publiques de l'Union Européens dans ses divers états membres fournit de nombreuses illustrations de ce problème (Smyrl, 2000).

\section{Changement de préférences et chan- gement de stratégie}

Dans le vocabulaire de la théorie des jeux, un changement de stratégie correspond à un choix différent dans le cadre d'un jeu donné. Un changement de préférence correspond à un changement de jeu. Dans une approche plus généraliste, nous pouvons distinguer entre un changement de question et un changement de réponse (à une question donnée) ou entre un changement de moyens et de fins (Hall, 1993).

Un changement de préférence implique, entre autre, une redéfinition effective des " intérêts " de l'acteur en question. Or, la possibilité d'un tel changement est exclue par définition dans les approches institutionnalistes. Tout changement de comportement doit être interprété comme simple changement de stratégie. L'approche par le politique permet d'élargir le champ de recherche, tout en conservant la puissance analytique de la théorie des jeux.

Un changement de jeu peut suivre l'introduction de nouveaux buts ou de nouveaux joueurs, d'un changement dans la valeur absolue (objective) des enjeux, ou encore, d'un changement de valeur relative des enjeux dans le jugement subjectif d'un acteur ${ }^{13}$. Ces possibilités servent d'autant de pistes à suivre quand nous observons un changement dans le comportement d'un acteur.
Il faut être attentif, bien évidemment, avant d'identifier un changement de préférence, à la possibilité que le jeu observé ne soit, en fait, qu'un élément stratégique d'un jeu plus vaste - et plus stable. Ceci dit, l'erreur inverse: attribuer à l'acteur un but ultime si flou et général que n'importe quelle action puisse être interprétée comme stratégie visant à l'accomplir, est encore plus redoutable - et bien plus répandue ${ }^{14}$.

\section{Les décisions non-rationnelles: erreurs de traduction et méta-choix}

C'est justement pour éviter l'erreur qui consiste à trop élargir la notion de rationalité que nous devons établir avec soin les conditions nécessaires à tout choix réellement "rationnel ". L'action pleinement rationnelle est celle qui suit un principe établi et cohérent, avec une chance raisonnable de réussite. Deux points de cette définition restrictive de la rationalité sont de premier intérêt pour l'étude des politiques publiques.

Le premier est celui de la " mauvaise traduction "d'un principe. Nous retrouvons cette dynamique dans de nombreux cas de mise en œuvre, quand les intentions de ceux qui ont élaboré la politique en question se trouvent mal comprises, ou retraduites, par ceux chargés de son exécution. Cette possibilité est particulièrement probable dans le cas, évoqué ci-dessus avec l'exemple des fonds structurels européens, où la mise en œuvre se déroule dans un milieu institutionnel différent de celui de l'élaboration.

Le second exemple de choix non-rationnel est très différent, car ici il n'y a pas de principe applicable du tout, soit qu'un choix multidimensionnel rende la maximisation linéaire impossible, soit que l'acteur appelé à choisir ne dispose pas des conditions suffi- 
santes pour faire un choix " rationnel " mais se trouve contraint de choisir quand même. L'analyse du processus de choix de l'Airbus militaire A400M en donne une illustration (Joana, Smith, 2004). Nous appelons une situation de ce genre "méta-choix " car le décideur doit avant tout faire le choix de comment choisir.

Dans le cas le plus simple, le problème se limite à un manque d'informations. Le joueur ignorant les véritables enjeux de la partie ou même les conséquences probables des stratégies à sa disposition, il peut être appelé à " jouer " plus ou moins au hasard. Même en possession d'informations adéquates, un décideur peut se trouver dans une situation nonrationnelle si le choix en question porte sur plusieurs dimensions incommensurables, rendant impossible l'exercice de maximisation globale cher aux rationalistes ${ }^{15}$.

Dans tous les cas de figures, un méta-choix est important s'il est " institutionnalisé ", pour former la base de choix " ordinaires " ultérieurs. Une autre façon de dire la même chose est qu'un meta-choix important marque un point de bifurcation dans une chaîne de path dependence.
Pour mettre l'accent sur la question du rôle du politique dans les politiques publiques, nous insisterons dans notre sélection d'études empiriques, tout comme dans notre discussion théorique, sur les trois aspects évoqués ci-dessus. Il ne suffit pas, de notre point de vue, de savoir "qui gouverne?" Il faut également poser les questions "comment?" et " pour quoi faire?" L'analyse des institutions apporte depuis vingt ans certaines réponses à ces questions. Notre ambition est d'en ajouter quelques autres. Notre apport, en privilégiant le politique, est de permettre une meilleure compréhension de la frontière entre structures et choix, et une analyse plus précise des conditions et des conséquences de ces choix.

Nous insistons également sur le sens du mot "politique " dans le langage quotidien, où " politique " est bien souvent l'inverse de " raisonnable ". Le politique dans le monde réel est bel et bien le domaine de l'incertitude, du compromis, des choix imparfaits.

\section{Notes}

1. Ont collaboré à cet article: William Genieys (CEPEL), Stephanie Golob (Baruch College, City University of New York), Jean Joana (CEPEL), Laura Michel (CEPEL), Andy Smith (CERVELVIPOF), Marc Smyrl (CEPEL).

2. C'est par ce binôme rationalité axiologique/vecteur organisationnel que l'institutionnalisme sociologique se démarque clairement, aussi bien de la notion wébérienne de la bureaucratie (qui met en avant la rationalité instrumentale) que du " modèle rationnel généralisé " de Raymond Boudon (2003) (qui fait de l'individu, et non de l'organisation, le vecteur de la rationalité axiologique).

3. Cette critique vaut, à notre avis, tout autant pour les rationalistes « classiques " tel que Tsebelis, pour qui les préférences sont établies par postulat, que pour les empiristes, comme les disciples de Michel Crozier, qui cherchent à les découvrir par observation.

4. On retrouve dans cette catégorie les travaux de March et Olsen, mais aussi ceux de Putnam et de Dobbins.

5. Kymlicka, Will, 1989. Le cas de Sandel est sans doute le plus intéressant des trois, car lui-même ne le reconnaît pas toujours. Il est quand même implicite dans certains exemples qu'il met en avant, tel celui de Robert E. Lee devant choisir, à la veille de la guerre de sécession, entre devoir aux États Unis ou à son état natal de Virginie ; bien qu'anti-esclavagiste de conviction, il choisit le second et devint général en chef des 
armées sudistes. (Sandel, Michael, Democracy's Discontents: America in Search of a Public Philosophy. Harvard University Press, 1996. pp. 15, 16).

6. Skocpol, 1992. Ce livre traite également de la politique familiale américaine du début du XX' siècle et d'un cas négatif: l'échec des premières tentatives pour développer une politique générale de protection des travailleurs. Le titre présente bien le problème: pourquoi les (anciens) soldats et les mères de famille - et eux seuls - ont-ils eu « droit » à une protection sociale dans l'Amérique d'avant 1930?

7. Cette formulation se rapproche également de la notion de " pouvoir de troisième ordre " proposée par un autre auteur néo-Gramscien, S. Lukes. La manipulation et le contrôle de l'idéologie dominante, pour Lukes, permettent à une élite de s'imposer sans avoir à remporter un conflit visible, (Lukes, 1974).

8. Nous évitons ici le vocable "médiateur " fréquent dans le discours de l'école française car nous y trouvons un double sens parfois troublant. Le " médiateur ", dans la grille d'analyse proposée ci-dessous, n'est pas l'initiateur ou l'auteur d'un nouveau référentiel ou d'une nouvelle politique publique, mais plutôt celui qui assure les opérations de " décodage " et " transcodage " permettant de mettre en relation les acteurs ne partageant pas un référentiel commun.

9. Nous insistons sur le fait que l'étendue d'un ensemble cognitif, tout comme le cadre qui le régit, est une construction intellectuelle et non une réalité concrète. Il faut donc s'attendre à voir cette étendue définie de manière différente selon l'époque et le contexte philosophique et social - et les frontières entre ensembles aussi vivement contestés qu'âprement défendus.

10. Waltzer, Michael, 1885. Dans cette œuvre philosophique, Walzer maintient que les principes de juste distribution ne sont pas les mêmes selon la question traitée - une l'échange d'un service contre payement en espèce, par exemple, est " juste " dans la " sphère " de l'économie marchande, mais ne le serait pas en politique... ou en amour. Par analogie, nous soutenons qu'une politique "logique ", i.e. conforme au cadre cognitif, dans l'ensemble économique, ne le serait peut-être pas dans l'ensemble militaire ou sanitaire (1985).

11. Le programme constitué par la lutte contre les stupéfiants, par exemple, appartient-il à l'ensemble sanitaire, celui du maintien de l'ordre public ou un autre encore (économie, défense nationale...) ? La légitimité accordée à un modèle programmatique donné (traitement, répression, etc.) dépendra en grande partie de la réponse à cette première question.

12. Certains aspects de la politique de l'armement, en particulier le choix entre cadre national ou européen relève de cette dynamique. Voir à titre d'exemple nos travaux sur les politiques d'armements avec des études de cas sur le char Leclerc et l'Airbus A400M (Joana, Smith, 2004; Genieys, Michel, 2004).

13. Ce dernier élément correspond à la notion " d'orientation d'interaction " introduite par Scharpf. Dans le contexte d'un jeu donné, le passage d'une orientation " égoïste ", consistant à maximiser ses propres gains sans référence aux résultats des autres joueurs, à une orientation " adversaire " où il faut, à tout prix, minimiser les gains de l'autre, doit être interprété comme changement de préférence.

14. Cette erreur, à notre avis, est typique du modèle de la " rationalité généralisée " de Boudon.

15. Nous retrouvons avec ce dernier exemple la notion de "l'hyper-choix " évoquée par Jobert et Muller. 


\section{Références}

Behrens P., Smyrl M., "A Conflict of Rationalities ", Journal of European Public Policy, vol. 6, n², 1999.

Boudon R., Raisons: Bonnes Raisons, Paris, Presses Universitaires de France, 2003.

DiMaggio P., Powell W., The New Institutionalism in Organizational Analysis, University of Chicago Press, 1991.

Dobbin F., Forging Industrial Policy, Cambridge, Cambridge University Press, 1994.

Faure A., Pollet G., Warin Ph., (eds.), La Construction du Sens dans les Politiques Publiques. Paris, l'Harmattan, 1995.

Genieys W., Hassenteufel P., "Entre les politiques publiques et la politique: l'émergence d'une élite du Welfare?", Revue Française des Affaires Sociales, $n^{\circ}$ 4, 2001.

Genieys W., "La constitution d'une élite du Welfare en France dans la France des années 90 ", Sociologie du travail, vol. $47, \mathrm{n}^{\circ} 2,2005$.

Genieys W., Michel L., "L'invention du meilleur char au monde: le Leclerc ", in W. Genieys (dir.), Le choix des armes. Théories, acteurs et politiques, Paris, CNRS éditions, 2004.

Hall P., " The movement from Keynesianism to monetarism : institutional analysis and British economic policy in the 1970s ", in Steinmo T., Longstreth (eds.), Structuring Politics: Historical Institutionalism in Comparative Analysis, Cambridge, Cambridge University Press, 1992.

Hall P., Taylor R., "La Science Politique et les Trois Néo-Institutionnalismes ", Revue Française de Science Politique, vol. 47, n 3-4, 1997.

Hall P., "Preference Formation as a Political Process: The Case of European Monetary Union », in Katznelson, Weingast (eds.). Preferences over Time. New York, Russel Sage Foundation, 2004.

Huber J., Rationalizing Parliament: Legislative Institutions and Party Politics in France, Cambridge and New York, Cambridge University Press, 1996.

Joana J., "La condition militaire: inventions et réinventions d'une catégorie d'action publique ", Revue Française de Science politique, vol. 52, $\mathrm{n}^{\circ} 4$, août 2002.

Joana J., "Les politiques de la ressource humaine des armes en France et en Grande-Bretagne: le sens des réformes ", Revue Française de Science Politique, vol. 54, n 5, octobre 2004.

Joana J., Smith A., "Le cas de l'avion de transport européen Airbus A400M. Politique inédite ou édifiante ", in W. Genieys (dir.), Le choix des armes, op. cit.

Jobert B., Muller P., L'État en Action: Politiques Publiques et Corporatismes, Paris, Presses Universitaires de France, 1987.

Jobert B., "Une approche dialectique des politiques publiques: l'héritage de l'État en Action ", Pôle Sud, $\mathrm{n}^{\circ} 21$, novembre 2004.

Katzenstein P., Cultural Norms and National Security: Police and Military in Post-War Japan, Ithaca, Cornell University Press, 1996.

Kymlicka W., Liberalism, Community and Culture, Oxford, Oxford University Press, 1989.

Lukes S., Power: A Radical View. London, MacMillan, 1974.

March J., Olsen J., Rediscovering Institutions, New York, The Free Press, 1989.

Mayntz, R., Scharpf F., "L'institutionalisme centre sur les acteurs ", Politix, n 55, 2001.

Meyer J., Rowan B., "Institutionalized Organizations: Formal Structure as Myth and Ceremony. "American Journal of Sociology, $\mathrm{n}^{\circ}$ 83, 1977.

Moravcsik A., The Choice for Europe, Ithaca, Cornell University Press, 1998.

Muller P., "Les politiques publiques comme construction d'un rapport au monde ", in Faure A. et al., La construction du sens dans les politiques publiques, Paris, l'Harmattan, 1995.

Muller P., "L'État en action revisité ", Pôle Sud, $n^{\circ} 21$, novembre 2004.

Muller P., " Esquisse d'une Théorie du Changement dans l'Action Publique „, Revue Française de Science Politique, 2005.

North, D., Robert T., The Rise of the Western World, Cambridge, Cambridge University Press, 1973.

North D., Institutions, Institutional Change, and Economic Performance, Cambridge, Cambridge University Press, 1990. 
Pierson P., "Increasing Returns, Path Dependence, and the Study of Politics ", American Political Science Review, vol. 94, $\mathrm{n}^{\circ} 2,2000$.

Pierson P., Politics in times. History, Institutions and social analysis, Princeton, Princeton University Press, 2004.

Putnam R., Making Democracy Work, Princeton, Princeton University Press, 1993.

Rawls J., Libéralisme Politique, Paris, PUF, 1993.

Sandel M., Democracy's Discontents: America in Search of a Public Philosophy, Harvard University Press, 1996. Scharpf F., Games Real Actors Play. Boulder, CO., Westview, 1997.

Skocpol Th., Protecting Soldiers and Mothers: The Political Origins of Social Policy in the United States, Cambridge MA, Belkamp Press of Harvard University Press, 1992.

Smyrl M., "When Ideas get in the Way of Reforms ", in Braun, Busch (eds.)., Public Policy and Political Ideas. Cheltenham, UK: Edward Elgar, 2000.

Smyrl M., "Politics et Policy dans les approches américaines des politiques publiques: Effets institutionnels et dynamique du changement ", Revue Française de Science Politique, vol. 52, nº 1, 2002.

Streeck, W., Thelen K (eds.), Institutional Change in Advanced Political Economies, Oxford, Oxford University Press, 2005.

Surel Y., "Idées, intérêts, institutions dans l'analyse des politiques publiques ", Pouvoirs, n 87, 1998.

Tsebelis G., Nested Games: Rational Choice in Comparative Politics, Berkeley, University of California Press, 1990.

Walzer M., Spheres of Justice, New York, Basic Books, 1984. 